\title{
Computed tomography in the diagnosis of malignant brain tumours: do all patients require biopsy?
}

\author{
M S CHOKSEY,* A VALENTINE, * H SHAWDON, $\dagger$ C E L FREER, $\ddagger$ K W LINDSAY* \\ From the Royal Free *and Charing Cross Hospitals, $\dagger$ London and Addenbrooke’s Hospital, Cambridge $\ddagger U K$
}

SUMMARY A proportion of patients with computed tomographic (CT) scan appearances of malignant brain tumour undergo conservative management, despite the absence of histological confirmation of the diagnosis. Concern that this policy risked misdiagnosing a benign tumour prompted us to examine the accuracy of CT scanning in diagnosing malignant lesions. The study was designed to determine whether within a group of 300 patients with intracerebral mass lesions of known pathology, two sub-groups existed: one with appearances so specific for malignant glioma that biopsy was unnecessary, and the other in which the appearances were characteristic of malignancy, though not specific for glioma. Three neuroradiologists independently reviewed the CT scans, together with brief clinical details. When diagnosing malignant tumours, all made errors: nine benign lesions were considered to be malignant. When diagnosing malignant glioma, one neuroradiologist made errors, but the other two adopted a more cautious approach and were accurate. The restricted a "certain" diagnosis to about one in five scans considered to show malignant tumour. Those diagnosed specifically as malignant glioma were intrinsic, irregular, mixed density lesions, exhibiting variable enhancement and infiltrating the peri-ventricular tissues, especially the corpus callosum. Using these criteria, they could correctly identify a small proportion of patients with malignant gliomas. In all other patients, biopsy remains the only means of obtaining a definitive diagnosis.

Malignant glioma carries a poor prognosis, irrespective of treatment.' A recent publication, ${ }^{2}$ confirmed anecdotal experience that many neurologists and neurosurgeons adopted a conservative approach in some patients with suspected malignant brain tumours, and did not submit them to biopsy for histological confirmation. In these patients, the diagnosis rested on clinical and radiological evidence, in particular computed tomography (CT). The authors implied that conservative management incurred less short term morbidity than in patients undergoing burr-hole biopsy. Concern that this conservative approach was potentially harmful led us to design a study, aimed at answering the following question: "Can you diagnose intracerebral malignancy on clinical and radiological groups alone, without running the risk of missing a benign lesion?"

Address for reprint requests: M S Choksey, Department of Neurosurgery, Newcastle General Hospital, Westgate Rd, Newcastle upon Tyne, NE4 6BE, UK.

Received 25 March 1988 and in revised form 2 December 1988. Accepted 1 January 1989
In 1979, Kendall et $a l^{3}$ outlined difficulties in diagnosing supratentorial glioma on CT scan appearances alone. The authors concluded that to make a firm diagnosis in every instance incurred a substantial risk of error, not only in failing to recognise a malignant lesion, but more importantly, in diagnosing a benign lesion as malignant. Whilst recognising that errors in CT scan diagnosis occur, we wondered whether a proportion of patients with a malignant brain tumour (primary or secondary) might exhibit CT scan appearances so typical that histological confirmation would become unnecessary. If so, the clinician could avoid biopsy with its inherent risks, without the fears of missing a remediable lesion. In this study, we examined whether within a group of patients with supratentorial mass lesions, two subgroups existed. One, in which neuroradiologists would correctly identify these as malignant tumours, and the second in which they could further identify these as malignant gliomas.

\section{Patients and methods}

The Royal Free Hospital, London, the Charing Cross 
Hospital, London, and Addenbrooke's, Cambridge, participated in the study. At each centre, we retrieved the records of 100 patients who had presented to the neurosurgical units with supratentorial mass lesions on CT scan, and had subsequently undergone biopsy through either burr hole or craniotomy. All lesions had been identified histologically. The patients underwent operation in the period 1979-1984. Most cases proved to be malignant glioma, but a wide range of pathology was encountered (table 1).

Table 1 Pathological diagnoses in 300 patients

\begin{tabular}{lc}
\hline Actual pathology & Number \\
\hline Malignant glioma & 207 \\
Secondary deposits & 50 \\
Abscess & 23 \\
Meningioma & 6 \\
Giant aneurysm & 3 \\
Encephalitis & 2 \\
Infarct & 3 \\
Tuberculosis & 2 \\
Haematoma & 1 \\
Epidermoid cyst & 1 \\
Arachnoid cyst & 1 \\
\hline
\end{tabular}

The CT scanner type varied from centre to centre. Of the 300 scans, 240 were performed on an EMI 1010 scanner, 46 on an EMI 7020 and 14 on a Siemens Somatom DR2.

Salient information from the patient's history and clinical examination accompanied each set of plain and enhanced scans (table 2). Each of three experienced neuroradiologists (AV, HS, and CF) reviewed the scans from the other two centres. (They had been in post for 6,22 and 4 years respectively). The 300 scans, reviewed twice, generated 600 sets of results. The neuroradiologists neither intercommunicated, nor had seen the scans previously.

The questionnaire presented to the neuroradiologists (table 3) determined whether they thought the lesion on the CT scan represented a "malignant glioma grade III/grade IV", a "malignant tumour" or "another lesion", and tested their degree of certainty. We described the diagnosis as radiologically "certain" when the neuroradiologists felt that a biopsy was unnecessary and "uncertain" when biopsy was required.

Table 2 shows the distribution of pathological diagnoses in the 300 patients. Of these 207 had malignant gliomas, 50 had metastases (most of which were solitary) and 23 had absces-

Table 2 An example of clinical details provided with each set of smears

\begin{tabular}{ll}
\hline Age 47 & On examination: drowsy \\
$6 / 52$ Headache & Left hemiparesis \\
2/52 Left-sided weakness & Left hemianopia \\
\hline
\end{tabular}

Table 3 Questionnaire that accompanied each set of scans

\begin{tabular}{lll}
\hline Does the CAT scan show: & & \\
A malignant glioma Grade 3-4? & Yes & No \\
If yes, is a biopsy needed to confirm the diagnosis? & Yes & No \\
A malignant tumour? & Yes & No \\
If yes, is a biopsy needed to confirm the diagnosis? & Yes & No \\
Other types of lesion?(Specify) & Yes & No \\
Lesion of uncertain pathology? & Yes & No \\
\hline
\end{tabular}

ses; in the remainder, other intracranial conditions simulated tumours. Each set of 100 scans contained a similar spread of material.

\section{Results}

Figure 1 shows the number of instances in which the neuroradiologist claimed to diagnose malignant gliomas with "certainty". These figures have been expressed both as absolute numbers and as percentages. For example, one neuroradiologist diagnosed 29 of the malignant gliomas presented to him with "certainty". As there were actually 137 histologicallyproven malignant gliomas among the 200 cases he reviewed, this represented $21 \%$. Similarly, fig 2 shows the number of instances in which they diagnosed malignant tumours other than gliomas. Marked variation occurred between the three neuroradiologists. Two appeared more cautious, making a confident diagnosis in only one in five instances. By contrast, the third made a confident diagnosis in more than two out of three.

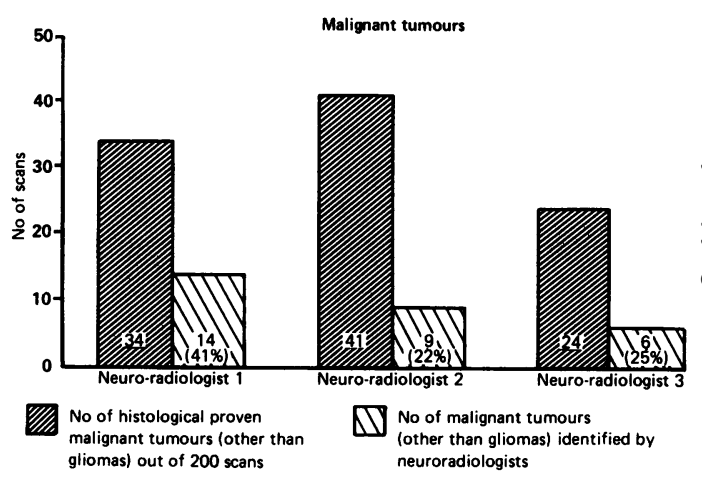

Fig 1 Diagram illustrating the level of "certainty" of the neuroradiologists in diagnosing malignant tumours (other than malignant gliomas).

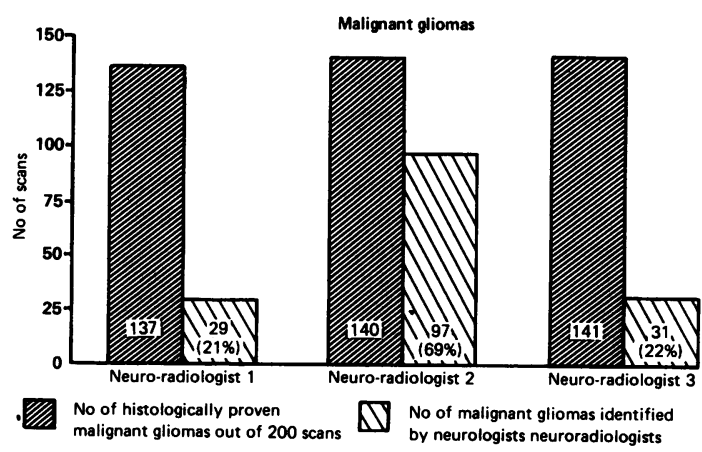

Fig 2 Diagram illustrating the level of "certainty" of the neuroradiologists in diagnosing malignant gliomas. 
Errors

All the neuroradiologists made errors, tabulated in tables 4 and 5. Overall, there were 12 errors out of 600 sets of results. This gives an apparent error rate of $12 / 600(2 \%)$. However, the true rate is actually higher, when expressed not as a percentage of the total number of scans reviewed, but as a percentage of the proportion in which a "certain" diagnosis was made. There were 254 instances of "certain" diagnosis. This gives an overall error rate of $12 / 254(4.7 \%)$. Nine of these errors were benign conditions which were diagnosed as malignant, (table 4) giving an error rate of $9 / 254(3.5 \%)$ in the diagnosis of malignancy.

Table 4 Errors where the neuroradiologist diagnosed a benign lesion as malignant

\begin{tabular}{ll}
\hline Actual diagnosis & Radiological diagnosis \\
\hline Abscess & Malignant glioma \\
Abscess & Malignant tumour \\
Giant aneurysm & Malignant glioma \\
Meningioma $\times 2$ & Malignant tumour \\
Infarct $\times 3$ & Malignant tumour \\
Encephalitis & Malignant tumour \\
\hline
\end{tabular}

Table 5 Errors when neuroradiologist diagnosed a malignant lesion as benign

\begin{tabular}{ll}
\hline Actual diagnosis & Radiological diagnosis \\
\hline Metastasis & Meningioma \\
Glioma & Meningioma \\
Encephalitis & Infarct \\
\hline
\end{tabular}

Included in these nine errors were two instances where an abscess and a giant aneurysm were each diagnosed as malignant glioma. However, the two neuroradiologists who were cautious about making a confident diagnosis of glioma did not make any errors in this particular subgroup. In addition to the nine errors, where benign lesions were identified as malignant, there were three other errors (table 5). In these, scans of a glioma and a metastasis were diagnosed as meningiomas, and a scan of herpes encephalitis was diagnosed as an infarct.

Of the 300 scans, 14 were performed on the more modern Siemens Somatom DR2 scanner. These generated 28 sets of data, which contained one major error-a patient with an infarct was diagnosed as having a metastasis. In only 11 out of $28 \mathrm{CT}$ scans could a radiologist make a "certain" diagnosis.

\section{Discussion}

In 1979, Kendall et al $^{3}$ highlighted the difficulties and pitfalls of diagnosing malignant gliomas on CT scan appearances alone. They noted that of 314 patients with tumours of known histology, $12.7 \%$ were misdiagnosed, and in $6.5 \%$ a benign lesion was incorrectly reported as malignant. Baker $e$ al $^{4}$ looked at the falsepositive and false-negative rate in the interpretation of nearly 3,000 scans. In their series, 13 out of $464(2 \cdot 8 \%)$ of non-neoplastic lesions were diagnosed as "neoplasms". In 80 of the $1071(7.5 \%)$ neoplasms, the neuroradiologists failed to diagnose the histological type.

Wroe $e a^{2}$ sought histological confirmation in 133 patients. In six they revised the pre-operative diagnosis. They used this to quote an error rate of $3 \%$ $(6 / 205)$. However, the correct denominator is 133 , not 205 , as they obtained histological proof in only the former group. This gives an error rate of $4 \cdot 5 \%(6 / 133)$. They gave no reason for these errors, not did they define their criteria for diagnosing malignancy on the CT appearances. In particular, they made no mention of the difficulties with peripheral lesions, in which the neuroradiologist may be unable to distinguish extraand intra-axial masses. In our study, two such lesions were mis-diagnosed: a giant aneurysm as a malignant glioma, and a glioma as a meningioma. They state confidently that no abscesses were misdiagnosed, but the remaining group of 72 patients may have harboured one. In the absence of confirmatory post mortem examination in all cases, this can neither be proven nor refuted.

In a study involving $89 \mathrm{CT}$ scans, McNeil et $\mathrm{ll}^{5}$ suggested that the provision of clinical information only marginally improved the accuracy of interpretation of CT scans, from $94 \%$ to $97 \%$ (Alternatively, this can be taken as halving the error rate.) In our study, we included a brief clinical history with each CT scan. Two of the three neuroradiologists thought it aided the radiological diagnoses.

Whilst accepting that diagnostic errors occur, in this study we have investigated the possibility that, in a proportion of patients, one can diagnose malignant tumours on clinical grounds and CT scan evidence alone, reserving biopsy for equivocal cases. Our findings show that this proportion, although small, does exist. Two of the three neuroradiologists adopted a cautious approach. When diagnosing malignant glioma, they committed themselves only when the tumour was clearly intrinsic, irregular, exhibited patchy enhancement and invaded the peri-ventricular tissues, especially the corpus callosum. By applying these criteria, they could correctly identify a sub-group of patients with malignant gliomas in whom biopsy was unnecessary. Significantly, this sub-group only accounted for approximately one fifth of the patients with malignant glioma and would therefore only reduce the number of biopsies required by a small margin. Despite this conservative approach, they too made errors, diagnosing benign lesions as malignant, although not as malignant gliomas.

One criticism of our study is that it was retrospec- 
tive, and that only patients referred to neurosurgical units were included. This could introduce a bias into the cohort; a greater than expected proportion of patients would have equivocal scan appearances. There is no simple answer to this; all we can do is point out that the alternative (and perhaps necessary) study would have to be prospective, and all patients diagnosed as having a malignant tumour would have to either be subjected to biopsy or necropsy. This would need the co-operation of neurologists, neurosurgeons, radiologists, pathologists and general practitioners. Of paramount importance would be the consent of all the deceased's relatives to a post-mortem examination, held for solely academic reasons. In our region, there are a number of ethnic minorities who would object strongly to this; unfortunately, these are just the patients who are likely to harbour potentially confusing pathology, such as abscesses, tuberculosis and even fungal disorders. The omission of even a few such patients would seriously undermine the results. In contrast, our study has one merit: a pathological diagnosis had been obtained for all the patients.

Primary lymphoma of the central nervous system usually appears as a hyperdense lesion on a plain CT scan, with homogenous enhancement after intravenous contrast injection. These tumours are less likely to be infiltrative, and are often multifocal. They were rare until the present AIDS epidemic, and our series did not contain any. We admit that our criteria could include them, and that in theory it is possible that such a tumour could be diagnosed as a malignant glioma, and not biopsied; however, this risk is extremely small. In addition, the prognosis in primary CNS lymphoma is poor; two series, by Hochberg et al and Ashby et al, ${ }^{67}$ quote median survival times of about one year, despite active treatment. Therefore, we feel that the small risk of missing a malignant lymphoma is justified.

Brain biopsy undoubtedly carries a risk, whether performed by craniotomy, trephine, needle biopsy, or stereotaxy. Marshall et $\mathrm{al}^{8}$ reported the results of needle biopsy in 53 patients with malignant astrocytoma; of these, two died $(3.9 \%)$. In both, conscious level had been depressed pre-operatively. Shetter $e a^{9}{ }^{9}$ compared the results of 20 freehand and 34 stereotactic biopsies of supratentorial masses. In the first group, no post operative deaths occurred within one week of biopsy; two patients had permanent neurological deficits. In the stereotactic group, one patient died postoperatively. Wroe et al have discussed the mortality and morbidity of biopsy, and we do not wish to reiterate the arguments. However, both papers quoted in favour of conservative management predate the widespread use of steroids and CT scanning, and the findings may no longer apply.

Any study which attempts to determine the true mortality of needle biopsy is hampered by the fact that many patients arrive at neurological centres in a rapidly deteriorating condition, and are submitted by biopsy to exclude a treatable lesion. On confirming malignancy, the rapid cessation of steroid therapy may precipitate early death. In these patients, it is unjustified to attribute all the post-operative mortality to the procedure alone. In contrast, the mortality of an undiagnosed abscess must approach $100 \%$. Our findings support the experience of many neurosurgeons, who have performed needle biopsies on what were ostensibly malignant gliomas, only to obtain pus. In this study, we have found an overall error rate of approximately $5 \%$. We accept that this may be in a highly-selected group, and that in more common clinical situations the rate may be lower. However, whether the risk is 1 in 50 , or 1 in 500 , we feel that no patient in this country should die of an undiagnosed cerebral abscess.

The three neuroradiologists participating in this study are experienced. All were aware that their reports were under close scrutiny and subject to histological confirmation, yet all made errors. We believe that such errors are inevitable, despite improvements in CT scanners. Our figures indicate that it is possible to achieve diagnostic certainty in only a small number of patients with malignant gliomas, and that improved image quality is unlikely to increase this proportion. Unequivocal but erroneous radiological reporting may lead the clinician to adopt a conservative approach. Without autopsy, such mistakes would remain undetected. In addition, conveying erroneous information of the expected prognosis to relatives or to the patient himself could cause unnecessary distress.

We conclude, however, that if neuroradiologists adhere strictly to the aforementioned criteria when diagnosing malignant gliomas, conservative management is justified, and would apply to approximately one in five patients. In all other patients, where the clinical situation demands it, biopsy remains the only means of obtaining a definitive diagnosis.

We thank Mr R S Maurice-Williams, Messrs J Gleave, A E Holmes and D G Hardy and Messrs R D Illingworth and $\mathrm{J} M$ Rice Edwards for allowing us to use their patients' records. We are grateful to Mrs Chandrika Shah and Miss Anna Grace for typing the manuscript.

This study was funded by the Royal Free Hospital Neurosurgical Research Grant G 275.

\section{References}

1 Saltzmann, M. Survival in glioblastoma: a historical perspective. Neurosurgery 1980;7:435-9. 
2 Wroe SJ, Foy PM, Shaw MDM, et al. Differences between neurological and neurosurgical approaches in the management of malignant brain tumours. $\mathrm{Br}$ Med J 1986;293:1015-8.

3 Kendall BE, Jakubowski J, Pullicino P, Symon L Difficulties in the diagnosis of supratentorial gliomas by CAT scan. J Neurol Neurosurg Psychiatry 1979;42: 485-92.

4 Baker LH, Houser OW, Campbell JK. National Cancer Institute study: evaluation of computed tomography in the diagnosis of intracranial neoplasms. Radiology 1980;136:91-6.

5 McNeil BJ, Hanley JA, Funkenstein HH, Wallman J. Paired receiver operating characteristic curves and the effect of history on radiographic interpretation. Radiology 1982;149:75-7.

6 Hochberg FH, Miller DC. Primary central nervous system lymphoma. J Neurosurg 1988;68:835-53.

7 Ashby MA, Bowen D, Bleehan NM, Barber PC, Freer CEL. Primary lymphoma of the central nervous system: experience at Addenbrooke's Hospital, Cambridge. Clin Radiol 1988;39:173-81.

8 Marshall LF, Jennett B, Langfitt TW. Needle biopsy for the diagnosis of malignant glioma. JAMA 1974; 228:1417-8.

9 Shetter AG, Bertuccini TV, Pitman HW. Closed needle biopsy in the diagnosis of intracranial mass lesions. Surg Neurol 1977;8:341-5. 